\title{
Pneumomediastinum and Subcutaneous Emphysema after Use of a High-Speed Dental Handpiece
}

\author{
You Kyeong Park, Chang Woo Choi, Hwa Kyun Shin \\ Department of Thoracic and Cardiovascular Surgery, Soonchunhyang University Bucheon Hospital, Bucheon, Korea
}

\begin{abstract}
Subcutaneous facial emphysema after dental treatment is an uncommon complication caused by dental procedures. However, in severe cases, it can spread to the neck, mediastinum, and thorax, resulting in pneumomediastinum and pneumothorax. Here we report two cases of subcutaneous emphysema and pneumomediastinum after dental treatment using an air-compressed, highspeed dental handpiece. We describe the diagnosis and treatment of iatrogenic pneumomediastinum.
\end{abstract}

Keywords: Pneumomediastinum; Mediastinal emphysema; Diagnostic imaging

\section{INTRODUCTION}

Here we report two cases of acute cervicofacial subcutaneous emphysema that extended to the mediastinum after dental treatment. These complications after dental treatment are rare and have a good prognosis. However, patients sometimes feel neck pain, discomfort when swallowing and embarrassed by swelling of the face. A dentist may also fail to take appropriate treatment early. Therefore I would like to discuss the course and treatment of this complication.

\section{CASE REPORTS}

\section{Case 1}

A 41-year-old female presented to the emergency room with acute facial swelling, difficulty swallowing, and chest pain with breathing. She had undergone dental treatment using an air-compressed high-speed dental handpiece in the mandibular right first molar (\#46) 1 hour previously. The patient had no past medical history, and her vital signs were stable: blood pressure of 130/80 $\mathrm{mm} \mathrm{Hg}$, heart rate of 76 , respiratory rate of 20, and temperature of $36.1^{\circ} \mathrm{C}$. Physical examination revealed right buccal, bilateral submandibular, and subclavicular swelling with crepitation. The de- partment of dental medicine was consulted, and the dentist revealed that the patient had no history of oral inflammation or signs of infection. Her white blood cell count was within normal limits. Neck anteroposterior and lateral radiographs showed extensive emphysema bilaterally along the neck and retropharyngeal space (Fig. 1). A chest radiograph showed pneumomediastinum and subcutaneous emphysema bilaterally along the lower neck. Facial and chest computed tomography (CT) showed pneumomediastinum with extensive emphysema along the cervicofacial soft tissue, lower neck, and chest wall (Fig. 2). Conservative treatment was performed, including no oral feeding, supplemental oxygen, intravenous amoxicillin and clavulanate, and daily oral irrigation. After 4 days, the swelling and crepitation gradually resolved and remained stable after returning to a normal diet. She was discharged on day 6 . Informed consent was provided by our patient for the publication of this case report.

\section{Case 2}

A 50-year-old female was referred with left facial and neck swelling. She had undergone dental treatment with an air-compressed high-speed dental handpiece in the mandibular left second premolar (\#35) 1 day previously. The patient had no past medical history, and physical examination revealed gross swelling and 


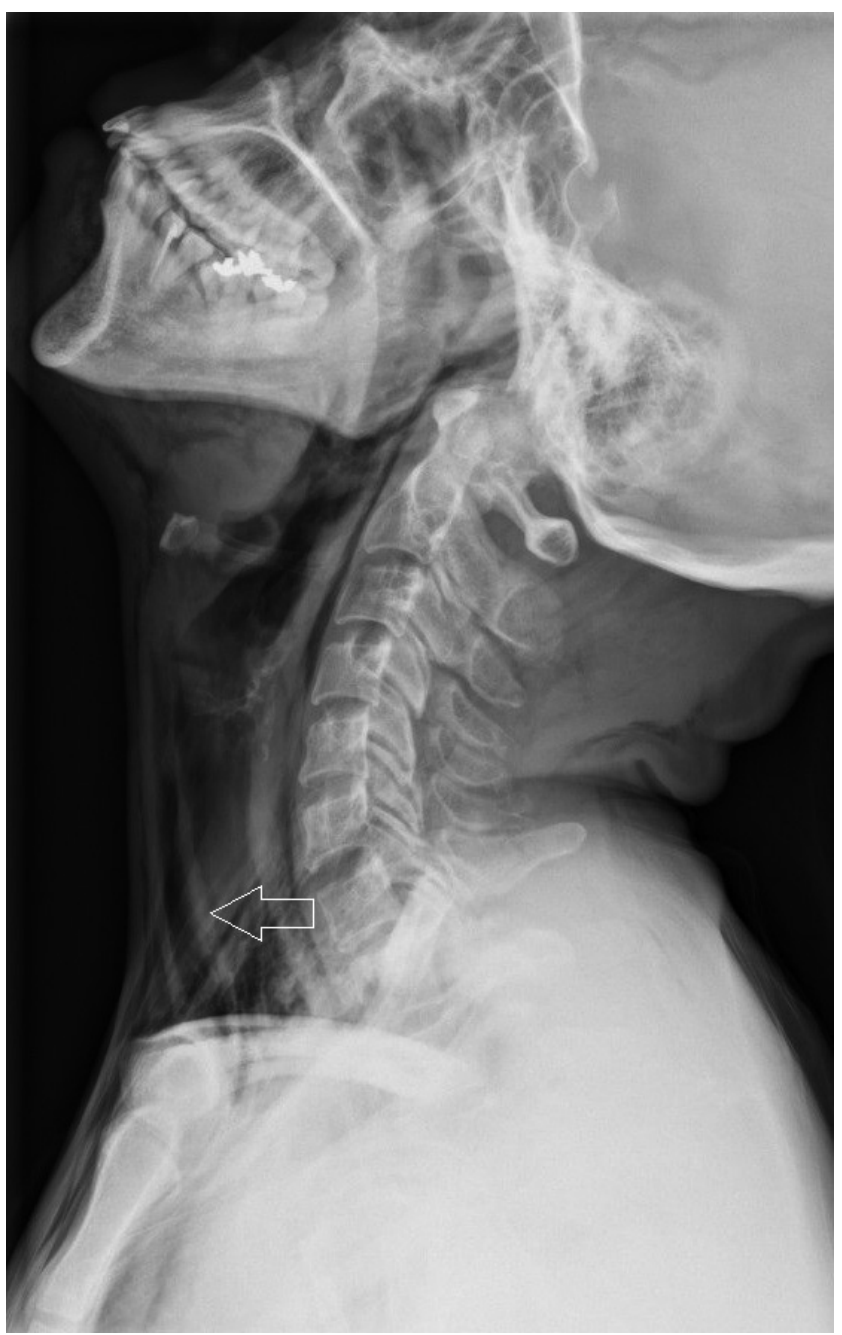

Fig. 1. Subcutaneous emphysema on neck lateral radiograph (arrow).

crepitation on the left side. Neck anteroposterior and lateral radiographs showed emphysema along the bilateral neck, and a chest radiograph showed pneumomediastinum in the upper thorax. Chest CT showed the same findings. She received the same conservative treatment mentioned above and the swelling and crepitation resolved after 3 days. She was discharged on day 6 . Informed consent was provided by our patient for the publication of this case report.

\section{DISCUSSION}

Pneumomediastinum is a condition in which air is present in the mediastinum. This can happen from physical trauma that damages the lungs or other situations that raise the pressure in the lungs, causing the alveoli to rupture. The most common symptom

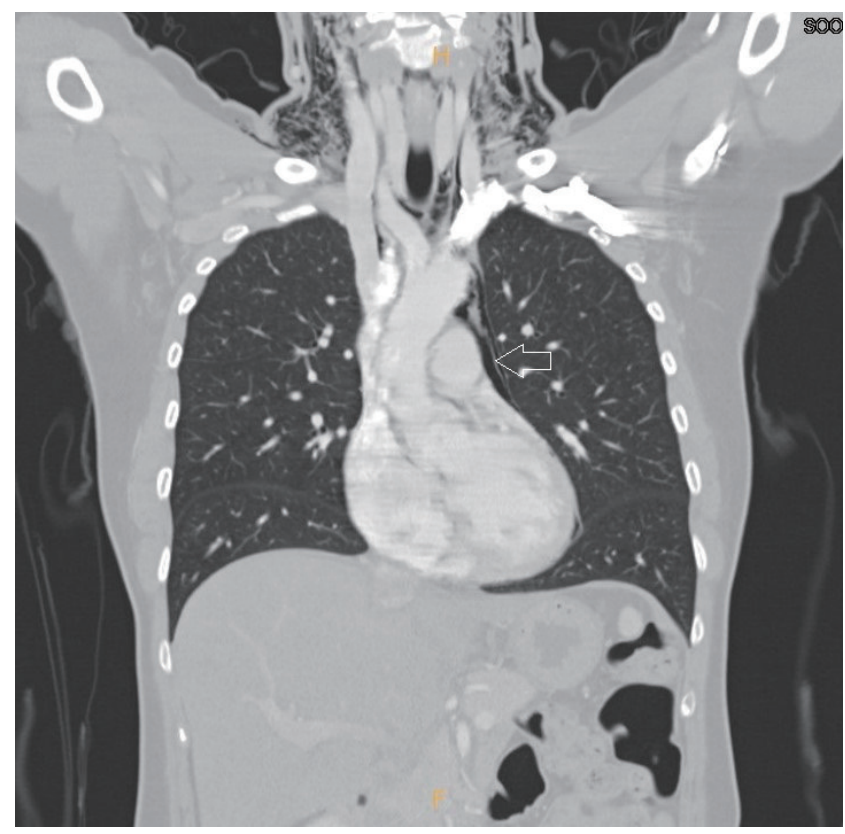

Fig. 2. Pneumomediastinum on chest computed tomography (arrow).

is central chest pain, and other symptoms include difficulty breathing, trouble swallowing, hoarse voice, and subcutaneous emphysema, specifically affecting the face, neck, and chest. Pneumomediastinum is generally considered a benign condition with a good prognosis. Treatment consists primarily of symptom relief. Oxygen inhalation can accelerate the reabsorption of air into the mediastinum. A rare complication is the accumulation of a significant amount of air in the mediastinum. In this case, pneumomediastinum can cause compression of mediastinal structures such as the coronary veins, leading to tension pneumomediastinum. Very rarely, air can leak into the pericardial space, causing pneumopericardium or even tamponade [1].

Subcutaneous facial emphysema after dental treatment is an uncommon complication caused by dental procedures. In severe cases, it can spread to the neck, mediastinum, and thorax, resulting in pneumomediastinum and pneumothorax [2]. Reported cases have all occurred following the use of air turbine drills for restorative treatment, extractions, root canal treatment, and periodontal treatment. Most cases occur after extraction of the mandibular third molar, in which the tooth is sectioned using an air turbine drill [3]. There are two pathways through which highpressure air invades subcutaneous tissue. One is through bone destruction caused by pathologic lesions or iatrogenic injury, and the other is through the periosteal and submucosal tissue [4]. If subcutaneous emphysema is suspected, the dental procedure should be 
stopped, and physical examination should be done, including imaging as necessary. If pneumomediastinum is diagnosed, the patient should be observed closely until the subcutaneous emphysema subsides. The use of empirical antibiotics to prevent secondary infection from oral flora is recommended if there are no contraindications. Supplemental oxygen can be useful as described above.

Our cases show that subcutaneous emphysema and pneumomediastinum after dental treatment using an air-compressed, high-speed dental handpiece can be treated effectively with conservative treatment unless the diagnosis is missed. Dentists should be careful when using a high-speed handpiece, and if the patient has a severe oral infection or iatrogenic injury, the dentist should closely observe the patient after dental care, especially when using the high-speed dental handpiece. Early diagnosis and proper con- servative management can treat them effectively.

\section{REFERENCES}

1. Herlan DB, Landreneau RJ, Ferson PF. Massive spontaneous subcutaneous emphysema: acute management with infraclavicular "blow holes". Chest 1992;102:503-5.

2. Chen SC, Lin FY, Chang KJ. Subcutaneous emphysema and pneumomediastinum after dental extraction. Am J Emerg Med 1999;17:678-80.

3. Arai I, Aoki T, Yamazaki H, Ota Y, Kaneko A. Pneumomediastinum and subcutaneous emphysema after dental extraction detected incidentally by regular medical checkup: a case report. Oral Surg Oral Med Oral Pathol Oral Radiol Endod 2009;107:e33-8.

4. Lee SW, Huh YH, Cha MS. Iatrogenic subcutaneous cervicofacial emphysema with pneumomediastinum after class V restoration. J Korean Assoc Oral Maxillofac Surg 2017;43:49-52. 